International Mathematical Forum, 2, 2007, no. 23, 1119 - 1128

\title{
Rough Marcinkiewicz Integrals On Product Spaces ${ }^{1}$
}

\author{
Ahmad Al-Salman \\ Department of Mathematics \\ Yarmouk University \\ Irbid, Jordan \\ alsalman@yu.edu.jo
}

\begin{abstract}
In this paper, we establish an $L^{p}$ boundedness result of a class of Marcinkiewicz integral operators on product domains with rough kernels.
\end{abstract}

Keywords: Marcinkiewicz integral operators, Fourier transform, Rough kernels

Mathematics Subject Classification: Primary 42B20; Secondary 42B15, 42B25

\section{Introduction}

Let $\mathbf{R}^{d}(d=n$ or $d=m), d \geq 2$ be the $d$-dimensional Euclidean space and $\mathbf{S}^{d-1}$ be the unit sphere in $\mathbf{R}^{d}$ equipped with the induced Lebesgue measure $d \sigma$. Suppose that $\Omega$ be a homogeneous function of degree zero on $\mathbf{R}^{n}$ which is integrable on $\mathbf{S}^{n-1}$ and $\int_{\mathbf{S}^{n-1}} \Omega(y) d \sigma(y)=0$. Then the Marcinkiewicz integral operator $\mu_{\Omega}$ which was introduced by E. M. Stein in ([20]) is defined by

$$
\mu_{\Omega}(f)(x)=\left(\left.\left.\int_{-\infty}^{\infty}\left|\int_{|y| \leq 2^{t}} f(x-y) \Omega(y)\right| y\right|^{1-n} d y\right|^{2} 2^{-2 t} d t\right)^{\frac{1}{2}} .
$$

E. M. Stein proved that if $\Omega \in \operatorname{Lip}_{\alpha}\left(\mathbf{S}^{n-1}\right),(0<\alpha \leq 1)$, then $\mu_{\Omega}$ is bounded on $L^{p}$ for all $1<p \leq 2$ ([20]). Subsequently, the study of the $L^{p}$ boundedness of $\mu_{\Omega}$ under various conditions on the function $\Omega$ has been studied by many authors ([2], [3], [4], [5], [7], [8], [9], [17], among others). A particular result that is closely related to our work is the boundedness result of $\mu_{\Omega}$ obtained by

\footnotetext{
${ }^{1}$ The publication of this paper is supported by Yarmouk university research council.
} 
Chen-Fan-Pan in ([9]). In fact, the authors of $([9])$ showed that $\mu_{\Omega}$ is bounded on $L^{p}$ for $p \in((2+2 \alpha) /(1+2 \alpha), 2+2 \alpha)$ provided that $\Omega$ satisfies the following condition

$$
\sup _{\xi \in \mathbf{S}^{n-1}} \int_{\mathbf{S}^{n-1}}\left|\Omega\left(y^{\prime}\right)\right|\left(\log \frac{1}{\xi \cdot y^{\prime}}\right)^{1+\alpha} d \sigma\left(y^{\prime}\right)<\infty,
$$

for some $\alpha>0$. The conditions (1.2) were introduced by Grafakos and Stefanov in their study of singular integral operators ([14]). It should be pointed out here that Grafakos and Stefanov showed that for any $\alpha>0$, the following relations hold:

$$
F\left(\alpha, \mathbf{S}^{n-1}\right) \nsubseteq L\left(\log ^{+} L\right)\left(\mathbf{S}^{n-1}\right) \text { and } L\left(\log ^{+} L\right)\left(\mathbf{S}^{n-1}\right) \nsubseteq F\left(\alpha, \mathbf{S}^{n-1}\right),
$$

where $F\left(\alpha, \mathbf{S}^{n-1}\right)$ is the space of all integrable functions on $\mathbf{S}^{n-1}$ which satisfy (1.2).

The main purpose of this paper is studying the $L^{p}$ boundedness of a class Marcinkiewicz integral operators on product domains. Namely, for suitable mappings $\varphi, \psi: \mathbf{R}^{+} \rightarrow \mathbf{R}$ and $\Omega \in L^{1}\left(\mathbf{S}^{n-1} \times \mathbf{S}^{m-1}\right)$ that satisfy the conditions

$$
\begin{aligned}
\Omega(t x, s y) & =\Omega(x, y) \text { for any } t, s>0 \text { and } \\
\int_{\mathbf{S}^{n-1}} \Omega(u, \cdot) d \sigma(u) & =\int_{\mathbf{S}^{m-1}} \Omega(\cdot, v) d \sigma(v)=0
\end{aligned}
$$

consider the operator $\mathcal{M}_{\Omega, \varphi, \psi}$ given by

$$
\mathcal{M}_{\Omega, \varphi, \psi} f(x, y)=\left(\int_{-\infty}^{\infty} \int_{-\infty}^{\infty}\left|F_{t, s}^{(\varphi, \psi)}(f)(x, y)\right|^{2} 2^{-2(t+s)} d t d s\right)^{\frac{1}{2}}
$$

where

$$
F_{t, s}^{(\varphi, \psi)}(f)(x, y)=\iint_{\Lambda(t, s)} f\left(x-\varphi(|u|) u^{\prime}, y-\psi(|v|) v^{\prime}\right)|u|^{1-n}|v|^{1-m} \Omega(u, v) d u d v
$$

and $\Lambda(t, s)=\left\{(u, v) \in \mathbf{R}^{n} \times \mathbf{R}^{m}:|u| \leq 2^{t}\right.$ and $\left.|v| \leq 2^{s}\right\}$.

Clearly, when $\varphi(r)=\psi(r)=r$, the operator $\mathcal{M}_{\Omega, \varphi, \psi}$ is the classical Marcinkiewicz integral operator on product domains which we shall denote by $\mathcal{M}_{\Omega, c}$. In ([16]), Ding showed that $\mathcal{M}_{\Omega, c}$ is bounded on $L^{2}$ provided that $\Omega \in L\left(\log ^{+} L\right)^{2}\left(\mathbf{S}^{n-1} \times \mathbf{S}^{m-1}\right)$. Subsequently, the authors of ([11]) proved the $L^{p}$ boundedness of $\mathcal{M}_{\Omega, c}$ for all $1<p<\infty$ under the condition $\Omega \in$ $L\left(\log ^{+} L\right)^{2}\left(\mathbf{S}^{n-1} \times \mathbf{S}^{m-1}\right)$. Recently, the $L^{p}$ boundedness of $\mathcal{M}_{\Omega, c}$ was established under the weaker condition $\Omega \in L\left(\log ^{+} L\right)\left(\mathbf{S}^{n-1} \times \mathbf{S}^{m-1}\right)$; see Choi $([15])$ for $p=2$ and Al-Qassem, Al-Salman, Chang, and Pan ([3]) for all $1<p<\infty$.

The primary focus of this paper is establishing $L^{p}$ estimates of $\mathcal{M}_{\Omega, \varphi, \psi}$ for various functions $\varphi$ and $\psi$, and for functions $\Omega$ satisfying conditions similar to 
(1.2). Our conditions on the function $\Omega$ are stated as follows:

$$
\sup _{\left(\xi^{\prime}, \eta^{\prime}\right) \in \mathbf{S}^{n-1} \times \mathbf{S}^{m-1}} \iint_{\mathbf{S}^{n-1} \times \mathbf{S}^{m-1}}\left|\Omega\left(u^{\prime}, v^{\prime}\right)\right|\left\{G\left(\xi^{\prime}, \eta^{\prime}\right)\right\}^{1+\alpha} d \sigma\left(u^{\prime}\right) d \sigma\left(v^{\prime}\right)<\infty,
$$

where $G\left(\xi^{\prime}, \eta^{\prime}\right)=\log ^{+}\left(\left|\xi^{\prime} \cdot u^{\prime}\right|^{-1}\right)+\log ^{+}\left(\left|\eta^{\prime} \cdot v^{\prime}\right|^{-1}\right)+\log ^{+}\left(\left|\xi^{\prime} \cdot u^{\prime}\right|^{-1}\right) \log ^{+}\left(\left|\eta^{\prime} \cdot v^{\prime}\right|^{-1}\right)$ for some $\alpha>0$.

For $\alpha>0$, we let $F\left(\alpha, \mathbf{S}^{n-1}, \mathbf{S}^{m-1}\right)$ be the space of all integrable functions on $\mathbf{S}^{n-1} \times \mathbf{S}^{m-1}$ which satisfy (1.8).

Clearly, $\bigcup_{q>1} L^{q}\left(\mathbf{S}^{n-1} \times \mathbf{S}^{m-1}\right) \subset F\left(\alpha, \mathbf{S}^{n-1}, \mathbf{S}^{m-1}\right)$ for any $\alpha>0$ and the inclusion is proper. Moreover, by (1.3) it can be easily seen that

$$
\begin{gathered}
F\left(\alpha, \mathbf{S}^{n-1}, \mathbf{S}^{m-1}\right) \nsubseteq L\left(\log ^{+} L\right)\left(\mathbf{S}^{n-1} \times \mathbf{S}^{m-1}\right) \text { and } \\
L\left(\log ^{+} L\right)\left(\mathbf{S}^{n-1} \times \mathbf{S}^{m-1}\right) \nsubseteq F\left(\alpha, \mathbf{S}^{n-1}, \mathbf{S}^{m-1}\right)
\end{gathered}
$$

Our results are the following:

Theorem 1.1. Suppose that $\varphi, \psi: \mathbf{R}^{+} \rightarrow \mathbf{R}$ are $\mathcal{C}^{2}$ increasing convex functions with $\varphi(0)=\psi(0)=0$. If $\Omega \in F\left(\alpha, \mathbf{S}^{n-1}, \mathbf{S}^{m-1}\right)$ for some $\alpha>0$ and satisfies (1.3)-(1.4), then $\mathcal{M}_{\Omega, \varphi, \psi}$ is bounded on $L^{p}\left(\mathbf{R}^{n} \times \mathbf{R}^{m}\right)$ for all $p \in$ $((2+2 \alpha) /(1+2 \alpha), 2+2 \alpha)$.

Theorem 1.2. Suppose that $\varphi, \psi: \mathbf{R}^{+} \rightarrow \mathbf{R}$ are $\mathcal{C}^{2}$ functions that satisfy

$$
\begin{gathered}
\left|\phi_{l}(t)\right| \leq C_{1, l} t^{d_{l}}, \quad\left|\phi_{l}^{\prime \prime}(t)\right| \leq C_{2, l} t^{d_{l}-2}, \\
C_{3, l} t^{d_{l}-1} \leq\left|\phi_{l}^{\prime}(t)\right| \leq C_{4, l} t^{d_{l}-1}
\end{gathered}
$$

for some $d_{l} \neq 0$ and $t \in(0, \infty)$, where $\phi_{1}=\varphi, \phi_{2}=\psi, C_{1, l}, C_{2, l}, C_{3, l}$, and $C_{4, l}$ are positive constants independent of $t$. If $\Omega \in F\left(\alpha, \mathbf{S}^{n-1}, \mathbf{S}^{m-1}\right)$ for some $\alpha>0$ and satisfies (1.3)-(1.4), then $\mathcal{M}_{\Omega, \varphi, \psi}$ is bounded on $L^{p}\left(\mathbf{R}^{n} \times \mathbf{R}^{m}\right)$ for all $p \in((2+2 \alpha) /(1+2 \alpha), 2+2 \alpha)$.

\section{Preparation}

We start this section by establishing certain Fourier transform estimates. For suitable mappings $\varphi, \psi: \mathbf{R}^{+} \rightarrow \mathbf{R}, \Omega \in L^{1}\left(\mathbf{S}^{n-1} \times \mathbf{S}^{m-1}\right)$, and positive real numbers $r_{1}$ and $r_{2}$, let $K_{\Omega, r_{1}, r_{2}}: \mathbf{R}^{n} \times \mathbf{R}^{m} \rightarrow \mathbf{R}$ be given by

$$
K_{\Omega, r_{1}, r_{2}}(\xi, \eta)=\iint_{\Gamma\left(r_{1}, r_{2}\right)} e^{-i\left\{\varphi(|x|) \xi \cdot x^{\prime}+\psi(|y|) \eta \cdot y^{\prime}\right\}}|x|^{1-n}|y|^{1-m} \Omega(x, y) d x d y
$$

where $\Gamma\left(r_{1}, r_{2}\right)=\left\{(x, y) \in \mathbf{R}^{n} \times \mathbf{R}^{m}: \frac{r_{1}}{2} \leq|x|<r_{1}, \frac{r_{2}}{2} \leq|y|<r_{2}\right\}$. 
We have the following:

Lemma 2.1. Suppose that $\Omega \in F\left(\alpha, \mathbf{S}^{n-1}, \mathbf{S}^{m-1}\right)$ for some $\alpha>0$ and satisfying (1.3)-(1.4).

(a) If $\varphi, \psi: \mathbf{R}^{+} \rightarrow \mathbf{R}$ are $\mathcal{C}^{2}$ increasing convex functions with $\varphi(0)=$ $\psi(0)=0$, then

$$
\begin{aligned}
\left|K_{\Omega, r_{1}, r_{2}}(\xi, \eta)\right| & \leq r_{1} r_{2} C\left|\varphi\left(r_{1}\right) \xi\right|\left|\psi\left(r_{2}\right) \eta\right| \\
\left|K_{\Omega, r_{1}, r_{2}}(\xi, \eta)\right| & \leq r_{1} r_{2} C\left(\log \left|\varphi\left(\frac{r_{1}}{2}\right) \xi\right|\right)^{-1-\alpha}\left(\log \left|\psi\left(\frac{r_{2}}{2}\right) \eta\right|\right)^{-1-\alpha} ; \\
\left|K_{\Omega, r_{1}, r_{2}}(\xi, \eta)\right| & \leq r_{1} r_{2} C\left|\varphi\left(r_{1}\right) \xi\right|\left(\log \left|\psi\left(\frac{r_{2}}{2}\right) \eta\right|\right)^{-1-\alpha} ; \\
\left|K_{\Omega, r_{1}, r_{2}}(\xi, \eta)\right| & \leq r_{1} r_{2} C\left(\log \left|\varphi\left(\frac{r_{1}}{2}\right) \xi\right|\right)^{-1-\alpha}\left|\psi\left(r_{2}\right) \eta\right| .
\end{aligned}
$$

(b) If $\varphi, \psi: \mathbf{R}^{+} \rightarrow \mathbf{R}$ are $\mathcal{C}^{2}$ functions that satisfy (1.10)-(1.11), then $K_{\Omega, r_{1}, r_{2}}$ satisfies (2.2)-(2.5) with $\varphi$ and $\psi$ in the right hand sides of (2.1)-(2.4) are replaced by the power functions $t^{d_{1}}$ and $t^{d_{2}}$ respectively where $d_{1}$ and $d_{2}$ are as in the statement of Theorem 1.2.

Proof. We shall start by the proof of (a). Let $I_{\Omega, r_{1}}: \mathbf{R}^{n} \times \mathbf{S}^{n-1} \rightarrow \mathbf{R}$ and $J_{\Omega, r_{2}}: \mathbf{R}^{m} \times \mathbf{S}^{m-1} \rightarrow \mathbf{R}$ be given by

$$
I_{\Omega, \varphi, r_{1}}\left(\xi, x^{\prime}\right)=\int_{1}^{2} e^{-i\left(\xi \cdot x^{\prime}\right) \varphi\left(\frac{r_{1} r}{2}\right)} d r \text { and } J_{\Omega, \psi, r_{2}}\left(\eta, y^{\prime}\right)=\int_{1}^{2} e^{-i\left(\eta \cdot y^{\prime}\right) \psi\left(\frac{r_{2} r}{2}\right)} d r .
$$

Then since $\varphi$ and $\psi$ are increasing functions, we immediately get

$$
\begin{aligned}
\left|I_{\Omega, \varphi, r_{1}}\left(\xi, x^{\prime}\right)-1\right| & \leq\left|\varphi\left(r_{1}\right) \xi\right| \\
\left|J_{\Omega, \psi, r_{2}}\left(\eta, y^{\prime}\right)-1\right| & \leq\left|\psi\left(r_{2}\right) \eta\right| .
\end{aligned}
$$

On the other hand since $\varphi$ and $\psi$ are convex increasing functions, by integration by parts we have

$$
\begin{aligned}
\left|I_{\Omega, \varphi, r_{1}}\left(\xi, x^{\prime}\right)\right| & \leq 2\left|\varphi\left(\frac{r_{1}}{2}\right) \xi \cdot x^{\prime}\right|^{-1} \\
\left|J_{\Omega, \psi, r_{2}}\left(\eta, y^{\prime}\right)\right| & \leq 2\left|\psi\left(\frac{r_{2}}{2}\right) \eta \cdot y^{\prime}\right|^{-1} .
\end{aligned}
$$

By combining the estimates $(2.9),(2.10)$, and the trivial estimates $\left|I_{\Omega, \varphi, r_{1}}\left(\xi, x^{\prime}\right)\right| \leq$ 1 and $\left|J_{\Omega, \psi, r_{2}}\left(\eta, y^{\prime}\right)\right| \leq 1$, we get

$$
\begin{aligned}
\left|I_{\Omega, \varphi, r_{1}}\left(\xi, x^{\prime}\right)\right| & \leq C\left(\log \left|\varphi\left(\frac{r_{1}}{2}\right) \xi\right|\right)^{-1-\alpha}\left\{\log \left|\xi^{\prime} \cdot x^{\prime}\right|^{-1}\right\}^{1+\alpha} \\
\left|J_{\Omega, \psi, r_{2}}\left(\eta, y^{\prime}\right)\right| & \leq C\left(\log \left|\psi\left(\frac{r_{2}}{2}\right) \eta\right|\right)^{-1-\alpha}\left\{\log \left|\eta^{\prime} \cdot y^{\prime}\right|^{-1}\right\}^{1+\alpha} .
\end{aligned}
$$

Now, we are ready to prove (2.2)-(2.5). By the cancelation property of $\Omega$ and the estimates (2.7) and (2.8), it is straightforward to see that (2.2) holds. To see that (2.3), notice that

$$
\left|K_{\Omega, r_{1}, r_{2}}(\xi, \eta)\right|
$$




$$
\leq r_{1} r_{2} C \iint \mathbf{S}^{n-1} \times \mathbf{S}^{m-1}\left|\Omega\left(x^{\prime}, y^{\prime}\right)\right|\left|I_{\Omega, \varphi, r_{1}}\left(\xi, x^{\prime}\right)\right|\left|J_{\Omega, \psi, r_{2}}\left(\eta, y^{\prime}\right)\right| d \sigma\left(x^{\prime}\right) d \sigma\left(y^{\prime}\right)
$$

when combined with the estimates (2.11)-(2.12) and the fact that $\Omega \in F\left(\alpha, \mathbf{S}^{n-1}, \mathbf{S}^{m-1}\right)$ imply $(2.3)$.

To see (2.4), notice that by the cancelation property of $\Omega$ in the $y^{\prime}$-variable, we have

$$
\begin{gathered}
\left|K_{\Omega, r_{1}, r_{2}}(\xi, \eta)\right| \\
\leq r_{1} r_{2} C \iiint_{\mathbf{S}^{n-1} \times \mathbf{S}^{m-1}}\left|\Omega\left(x^{\prime}, y^{\prime}\right)\right|\left|I_{\Omega, \varphi, r_{1}}\left(\xi, x^{\prime}\right)\right|\left|J_{\Omega, \psi, r_{2}}\left(\eta, y^{\prime}\right)-1\right| d \sigma\left(x^{\prime}\right) d \sigma\left(y^{\prime}\right) .
\end{gathered}
$$

Therefore, (2.4) follows by (2.14), (2.12), (2.7), and the fact that $\Omega \in F\left(\alpha, \mathbf{S}^{n-1}, \mathbf{S}^{m-1}\right)$. Finally, (2.5) follows by symmetry. This completes the proof of part (a).

To proof part (b), we claim that

$$
\begin{gathered}
\left|I_{\Omega, \varphi, r_{1}}\left(\xi, x^{\prime}\right)-1\right| \leq C\left|\left(r_{1}\right)^{d_{1}} \xi\right| \\
\left|J_{\Omega, \psi, r_{2}}\left(\eta, y^{\prime}\right)-1\right| \leq C\left|\left(r_{2}\right)^{d_{2}} \eta\right| . \\
\left|I_{\Omega, \varphi, r_{1}}\left(\xi, x^{\prime}\right)\right| \leq C\left|\left(r_{1}\right)^{d_{1}} \xi \cdot x^{\prime}\right|^{-1} \\
\left|J_{\Omega, \psi, r_{2}}\left(\eta, y^{\prime}\right)\right| \leq C\left|\left(r_{2}\right)^{d_{2}} \eta \cdot y^{\prime}\right|^{-1} .
\end{gathered}
$$

Clearly, the estimates (2.15)-(2.16) hold. The estimates (2.17)-(2.18) follow from the properties of the functions $\varphi$ and $\psi$ and an integration by parts (see ). Hence, the proof of part (b) is complete by (2.15)-(2.18) and repeating exactly the same argument in the proof of part (a). This completes the proof of part (b) and hence the lemma.

Now, we state the following lemma on maximal functions which can be prove by a well known bootstrapping argument ([13], [9]):

Lemma 2.2. For $\xi \in \mathbf{R}^{d}(d \geq 1)$ and a suitable function $\phi: \mathbf{R}^{+} \rightarrow \mathbf{R}$, let $M_{\xi, \phi}$ be the maximal function defined on $\mathbf{R}^{d}$ by

$$
M_{\phi, \xi}(f)(x)=\sup _{t \in \mathbf{R}}\left|2^{-t} \int_{0}^{2^{t}} f(x-\varphi(r) \xi) d r\right| .
$$

If $\phi$ is $\mathcal{C}^{2}$ increasing convex function with $\phi(0)=0$ or $\phi$ is $\mathcal{C}^{2}$ function satisfying the conditions (1.10)-(1.11), then

$$
\left\|M_{\phi, \xi}(f)\right\|_{p} \leq C\|f\|_{p}
$$

for all $1<p<\infty$ where $C$ independent of $\xi \in \mathbf{R}^{d}$. 
Now, we have the following:

Lemma 2.3. Suppose that $\Omega \in L^{1}\left(\mathbf{S}^{n-1} \times \mathbf{S}^{m-1}\right)$ and $\varphi, \psi: \mathbf{R}^{+} \rightarrow \mathbf{R}$ are suitable functions. Let $M_{\Omega, \varphi, \psi}$ be the maximal function defined on $\mathbf{R}^{n} \times \mathbf{R}^{m}$ by

$$
\begin{gathered}
M_{\Omega, \varphi, \psi} f(x, y) \\
=\left.\sup _{t \in \mathbf{R}} \frac{1}{2^{t+s}}\left|\iint_{\Lambda(t, s)} f\left(x-\varphi(|u|) u^{\prime}, y-\psi(|v|) v^{\prime}\right)\right| u\right|^{1-n}|v|^{1-m} \Omega(u, v) d u d v \mid .
\end{gathered}
$$

If $\varphi$ and $\psi$ are $\mathcal{C}^{2}$ increasing convex functions with $\varphi(0)=\psi(0)=0$ or $\varphi$ and $\psi$ are $\mathcal{C}^{2}$ functions satisfying the conditions (1.10)-(1.11), then

$$
\left\|M_{\Omega, \varphi, \psi} f\right\|_{p} \leq C\|f\|_{p}
$$

for all $1<p<\infty$.

Proof. Notice that by Hölder's inequality, we have

$$
\left\|M_{\Omega, \varphi, \psi} f\right\|_{p} \leq\|\Omega\|_{1} \iint_{\mathbf{S}^{n-1} \times \mathbf{S}^{m-1}}\left\|M_{\varphi, u^{\prime}}^{1} \circ M_{\psi, v^{\prime}}^{2} f\right\|_{p} d \sigma\left(u^{\prime}\right) d \sigma\left(v^{\prime}\right)
$$

where $M_{\varphi, u^{\prime}}^{1} f(x, y)=M_{\varphi, u^{\prime}} f(\cdot, y)(x), M_{\psi, v^{\prime}}^{2} f(x, y)=M_{\psi, v^{\prime}} f(x, \cdot)(y)$, and $\circ$ denotes the composition of operators. Hence the result follows by (2.20) and Lemma 2.2. This completes the proof.

\section{Proof of Main Results}

Proof of Theorem 1.1. For $t, s \in \mathbf{R}$, let $\sigma_{t, s}$ be the measure defined on the Fourier transform side by

$$
\hat{\sigma}_{t, s}(\xi, \eta)=2^{-(t+s)} K_{\Omega, 2^{t}, 2^{s}}(\xi, \eta)
$$

where $K_{\Omega, 2^{t}, 2^{s}}$ is given by (2.1). Then it follows that

$$
\mathcal{M}_{\Omega, \varphi, \psi}(f)(x, y)=\left(\int_{-\infty}^{\infty} \int_{-\infty}^{\infty}\left|\sigma_{t, s} * f(x, y)\right|^{2} d t d s\right)^{\frac{1}{2}} .
$$

By an elementary procedure, choose two collections of $\mathcal{C}^{\infty}$ functions $\left\{\omega_{k}^{(1)}\right\}_{k \in \mathbf{Z}}$ and $\left\{\omega_{k}^{(2)}\right\}_{k \in \mathbf{Z}}$ on $(0, \infty)$ with the following properties:

$$
\begin{aligned}
\operatorname{supp}\left(\omega_{k}^{(1)}\right) & \subseteq\left[\frac{1}{\varphi\left(2^{k+1}\right)}, \frac{1}{\varphi\left(2^{k-1}\right)}\right] \text { and } \operatorname{supp}\left(\omega_{k}^{(2)}\right) \subseteq\left[\frac{1}{\psi\left(2^{k+1}\right)}, \frac{1}{\psi\left(2^{k-1}\right)}\right] ; \\
0 & \leq \omega_{k}^{(1)}, \omega_{k}^{(2)} \leq 1 \\
\sum_{k \in \mathbf{Z}} \omega_{k}^{(1)}(u) & =\sum_{k \in \mathbf{Z}} \omega_{k}^{(2)}(u)=1
\end{aligned}
$$




$$
\left|\frac{d^{l} \omega_{k}^{(1)}}{d u^{l}}(u)\right|,\left|\frac{d^{l} \omega_{k}^{(2)}}{d u^{l}}(u)\right| \leq C_{l} u^{-l} .
$$

Define the functions $\left\{v_{k}^{(1)}: k \in \mathbf{Z}\right\}$ on $\mathbf{R}^{n}$ and $\left\{v_{k}^{(2)}: k \in \mathbf{Z}\right\}$ on $\mathbf{R}^{m}$ by

$$
\left(v _ { k } ^ { ( 1 ) } \hat { ) } ( x ) = \omega _ { k } ^ { ( 1 ) } ( | x | ^ { 2 } ) \text { and } \left(v_{k}^{(2)} \hat{)}(y)=\omega_{k}^{(2)}\left(|y|^{2}\right) .\right.\right.
$$

Therefore, by (3.2) and (3.5), we get

$$
\mathcal{M}_{\Omega, \varphi, \psi}(f)(x, y) \leq \sum_{k \in \mathbf{Z}} \sum_{j \in \mathbf{Z}} I_{k, j}(f)(x, y),
$$

where

$$
I_{k, j}(f)(x, y)=\left(\int_{-\infty}^{\infty} \int_{-\infty}^{\infty} \mid\left(\left.v_{\lfloor t\rfloor+k}^{(1)} \otimes\left(v_{\lfloor s\rfloor+j}^{(2)}\right) * \sigma_{t, s} * f(x, y)\right|^{2} d t d s\right)^{\frac{1}{2}}\right.
$$

Here, $\lfloor x\rfloor$ is the greatest integer function less than or equal to $x$. For $k, j \in \mathbf{Z}$, let $S_{k, j}$ be the operator given by

$$
S_{k, j} f(x, y)=\left(\int_{-\infty}^{\infty} \mid\left(\left.v_{\lfloor t\rfloor+k}^{(1)} \otimes\left(v_{\lfloor s\rfloor+j}^{(2)}\right) * f(x, y)\right|^{2} d t d s\right)^{\frac{1}{2}} .\right.
$$

Then, by an argument of Fefferman and Stein ([18]), it can be easily shown that

$$
\left\|S_{k, j}(f)\right\|_{p} \leq C\|f\|_{p}
$$

for all $p \in(1, \infty)$ with constant $C$ depends only on $p$ and the dimension of the underlying space $\mathbf{R}^{n} \times \mathbf{R}^{m}$.

By (3.10), Lemma 2.3, the fact that $\left\|\sigma_{t, s}\right\| \leq 1$, and the proof of Lemma 1 in $([13])$ with minor modification, we obtain the crude estimate

$$
\left\|I_{k, j}(f)\right\|_{p} \leq C\|f\|_{p}
$$

for all $p \in(1, \infty)$.

Now, we estimate $\left\|I_{k, j}(f)\right\|_{2}$. For $k, j \in \mathbf{Z}$, nonzero $\xi \in \mathbf{R}^{n}$, and nonzero $\eta \in \mathbf{R}^{m}$, let $E_{k}^{1}(\xi)$ and $E_{j}^{2}(\eta)$ be the intervals in $\mathbf{R}$ given by

$$
\begin{aligned}
& E_{k}^{1}(\xi)=\left[\log _{2}\left(2^{k-1} \varphi^{-1}\left(|\xi|^{-1}\right)\right), \log _{2}\left(2^{k+2} \varphi^{-1}\left(|\xi|^{-1}\right)\right)\right] \\
& E_{j}^{2}(\eta)=\left[\log _{2}\left(2^{k-1} \psi^{-1}\left(|\eta|^{-1}\right)\right), \log _{2}\left(2^{k+2} \psi^{-1}\left(|\eta|^{-1}\right)\right)\right] .
\end{aligned}
$$

Clearly, we have the following

$$
\begin{aligned}
\left|E_{k}^{1}(\xi)\right| & =\left|E_{j}^{2}(\eta)\right|=3 \\
\varphi\left(2^{-k-1} \varphi^{-1}\left(|\xi|^{-1}\right)\right) & \leq \varphi\left(2^{t}\right) \leq \varphi\left(2^{-k+2} \varphi^{-1}\left(|\xi|^{-1}\right)\right) \\
\psi\left(2^{-j-1} \psi^{-1}\left(|\eta|^{-1}\right)\right) & \leq \psi\left(2^{s}\right) \leq \psi\left(2^{-j+2} \psi^{-1}\left(|\eta|^{-1}\right)\right)
\end{aligned}
$$


for $k, j \in \mathbf{Z}$, nonzero $\xi \in \mathbf{R}^{n}$, nonzero $\eta \in \mathbf{R}^{m}$, and $(t, s) \in E_{k}^{1}(\xi) \times E_{j}^{2}(\eta)$.

Therefore, Since the functions $\varphi$ and $\psi$ are convex increasing, the inequalities (3.13)-(3.14) imply that

$$
\begin{aligned}
\varphi\left(2^{t}\right) & \leq 2^{-k+2}|\xi|^{-1} \text { for } k \geq 3 \text { and } t \in E_{k}^{1}(\xi) \\
\varphi\left(2^{t-1}\right) & \geq 2^{-k+2}|\xi|^{-1} \text { for } k \leq-2 \text { and } t \in E_{k}^{1}(\xi) \\
\psi\left(2^{s}\right) & \leq 2^{-j+2}|\eta|^{-1} \text { for } j \geq 3 \text { and } s \in E_{j}^{2}(\eta) ; \\
\psi\left(2^{s-1}\right) & \geq 2^{-j+2}|\eta|^{-1} \text { for } j \leq-2 \text { and } s \in E_{j}^{2}(\eta) .
\end{aligned}
$$

Thus, by (2.2)-(2.5), (3.15)-(3.18), the trivial estimate $\left\|\sigma_{t, s}\right\| \leq 1$ if necessary, and Plancherel's theorem, we obtain

$$
\left\|I_{k, j}(f)\right\|_{2} \leq C_{k, j}\|f\|_{2}
$$

where

$$
C_{k, j}=\left\{\begin{array}{cc}
2^{-k-j} & , \text { if } k, j \geq 3 \\
2^{-k}|j|^{-(1+\alpha)} & , \text { if } k \geq 3 \text { and } j \leq-2 \\
2^{-j}|k|^{-(1+\alpha)} & , \text { if } j \geq 3 \text { and } k \leq-2 \\
|k j|^{-(1+\alpha)} & , \text { if } k, j \leq-2 \\
1 & , \text { if }-2 \leq k, j \leq 3 .
\end{array}\right.
$$

Hence, the result follows by (3.7) and an interpolation between (3.19) and (3.11) with any $p$.

Proof of Theorem 1.2. It can be easily seen that the proof of Theorem 1.2 follows by repeating exactly the same argument in the proof of Theorem 1.1 with minor modifications. We omit the details.

\section{References}

[1] H. Al-Qassem and Y. Pan, $L^{p}$ boundedness for singular integrals with rough kernels on product domains, Hokkaido Mathematical Journal 31 (2002), 555-613.

[2] H. Al-Qassem and A. Al-Salman, Rough Marcinkiewicz integral operators, International J. of Mathematics and Mathematical Sciences 27:8: 495-503.

[3] H. Al-Qassem, A. Al-Salman, L. Cheng, and Y. Pan, Marcinkiewicz integrals on product domains, Stud. Math. 167, No. 3, 227-234 (2005).

[4] Al-Salman, A. Marcinkiewicz functions along flat surfaces with hardy space kernels, J. Integral Equations Appl. 17 (4) (2005), 357-373.

[5] Al-Salman, A., On marcinkiewicz integrals along flat surfaces, Turk. J. Math., 29, (2005), 111-120. 
[6] A. Al-Salman and Y. Pan, Singular integrals with rough kernels, Can. Math. Bull. Vol. 47 (1), 2004 pp. 3-11.

[7] A. Al-Salman and H. Al-Qassem, Integral operators of Marcinkiewicz type, J. Integral Equations Appl. 14 (4) (2002).

[8] A. Benedek, A. Calderón and R. Panzone, Convolution operators on Banach space valued functions, Proc. Nat. Acad. Sci. U.S.A. 48 (1962) 356365 .

[9] J. Chen, D. Fan, and Y. Pan, A note on a Marcinkiewicz integral operator, Math. Nachr., 227, 33-42 (2001).

[10] J. Chen, D. Fan, Y. Pan, and Y. Ying, The method of rotation and Marcinkiewicz integrals on product domains, Studia Math. 153 (2002), 41-58.

[11] J. Chen, D. Fan, and Y. Ying, Rough Marcinkiewicz integrals with $L(\log L)^{2}$ kernels, Adv. Math. (China) 30 (2001), 179-181.

[12] J. Duoandikoetxea, Multiple singular integrals and maximal functions along hypersurfaces, Ann. Ins. Fourier (Grenoble) 36 (1986) 185-206.

[13] J. Duoandikoetxea and J. L. Rubio de Francia, Maximal functions and singular integral operators via Fourier transform estimates, Invent. Math. 84 (1986), 541-561.

[14] L. Grafakos and A. Stefanov, $L^{p}$ bounds for singular integrals and maximal singular integrals with rough kernel, India. Univ. Math. J., 47 (1998), 455-469.

[15] Y. Choi, Marcinkiewicz integrals with rough homogeneous kernel of degree zero in product domains, J. Math. Appl. 261 (2001), 53-60.

[16] Y. Ding, L2-boundedness of Marcinkiewicz integral with rough kernel, Hokkaido Mathematical Journal, 27 (1998), 105-115.

[17] Y. Ding, D. Fan, and Y. Pan, On the $\mathrm{L}^{p}$ boundedness of Marcinkiewicz integrals, Mich. Math. J., to appear.

[18] R. Fefferman and E. M. Stein, Singular integrals on product spaces,Adv. in Math. 45 (1982), 117-1143.

[19] D. Fan and Y. Pan, Singular integral operators with rough kernels supported by subvarieties, Amer. J. Math., 119 (1997),799-839. 
[20] E. M. Stein, On the functions of Littlewood-Paley, Lusin and Marcinkiewicz, Trans. Amer. Math. Soc. 88 (1958), 430-466.

[21] E. M. Stein, Harmonic Analysis: Real-Variable Mathods, Orthogonality and Oscillatory integrals, Princeton University Press, Princeton, NJ, 1993.

Received: January 23, 2006 